\title{
Optimal Broadcast Strategy-Based Producer Mobility Support Scheme for Named Data Networking
}

\author{
https://doi.org/10.3991/ijim.v13i04.10513 \\ Muktar Hussaini ${ }^{\bowtie}$, Shahrudin Awang Nor, Amran Ahmad \\ Universiti Utara Malaysia, Kedah, Malaysia \\ mail4.jrnl@gmail.com
}

\begin{abstract}
Named Data Networking is a consumer-driven network that supports content consumer mobility due to the nature of in-network catching. The catching suppressed unnecessary Interest packets losses by providing an immediate copy of the data and consumer-driven nature influencedthe mobile consumer to resend unsatisfied Interest packet immediately after the handoff. Once the producer moves to a new location, the name prefix changed automatically after handoff to the new router or point of attachment. The entire network lacks the knowledge of producer movement unless if the producer announces its new prefix to update the FIBs of intermediate routers. Lack of producer's movement knowledge causes an increase of handoff latency, signaling overhead cost, Interests packets losses, poor utilization of bandwidth and packets delivery. Therefore, there is needs to provide substantial producer mobility support to minimize the handoff latency, handoff signaling overhead cost, reduce the unnecessary Interest packets loss to improve data packets delivery once a content producer relocated. In this paper, broadcasting strategy is introduced to facilitate the handoff procedures and update the intermediate routers about the producer movement. Hence, analytical investigation result of this paper addresses the deficiency of Kite scheme by minimizing handoff signaling cost and provides data path optimization after the handoff.
\end{abstract}

Keywords - Network analysis model, signaling cost, handoff latency, Named data networking, Producer mobility support, broadcast strategy, mobility Interest

\section{Introduction}

Named Data Networking architecture is completely clean-slate architecture that evolved from the IP Internet architecture retain and expand end-to-end principle with routing and forwarding plane separation [1], [2]. The architecture of NDN maintained the IP hourglass architecture with the replacement of a thin waist with hierarchical named content in place of IP addresses. NDN focus on the name of the data and do away with the location of data [3]. The names were structured as hierarchical form and self-certifying with a digital signature. Moreover, the packets route by name discourses some problems pose in IP: address management, address space exhaustion, mobility that requires changing address and NAT traversal [1], [4]. Unlike in IP 
architecture that secured the connection from source to destination, NDN secured the content for integrity, authenticity, and confidentiality of data. The architecture comprises three nodes, consumer, producer, and routers. In addition, two packets Interest and Data (Fig. 1) were managed by three data structures Content Store (CS), Forwarding Information Base (FIB) and Pending Interest Table (PIT) to enable the named based routing[5], [6].
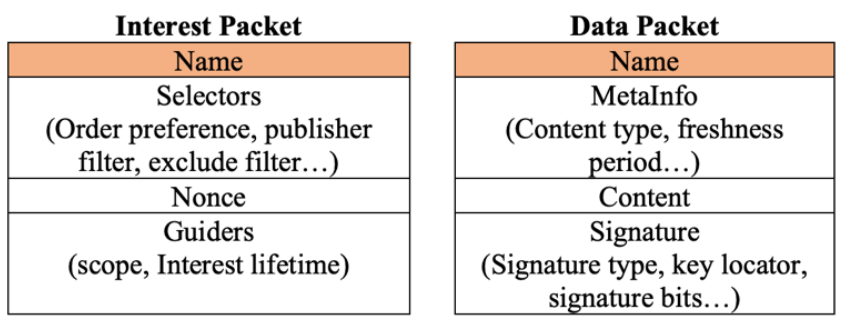

Fig. 1. NDN Data and Interest Format

NDN is a consumer-driven network that communications initiated from the interest of the consumer. A consumer sends and Interest that carries content prefix or name for the deification of desired data. A router that receives the Interest look up the CS for content availability, if found, the router replied back with data packets to the consumer, otherwise, the router recorded the interface of incoming Interest in PIT, then look up FIB for the information about the content populated by NBR protocol and forward the Interest further (Fig. 2). When the Interest reaches any router or content producer that has requested data, a data is sent back with name, data content and producer's signature via the path recorded in the PIT to the consumer. The data packet looks up the PIT, save in CS and forward downstream.

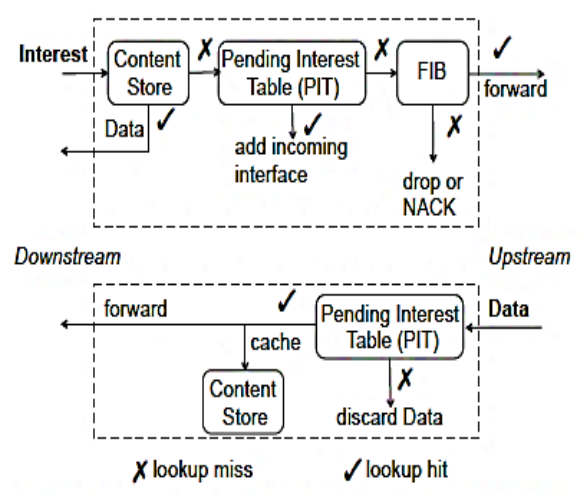

Fig. 2. Data Forwarding Process in NDN [1]

Among the Information-Centric Networking (ICN) approaches, Named Data Networking is the most recent approach; infrastructures less network architecture that provides network scalability due to its nature of hierarchical naming[7] [39]. NDN is 
hope and promising architecture for the future Internet that has been used and applied in the differentfields, such as Space-Terrestrial Integrated Network [8]WSN, Vehicular Network[9], [10] and real-time application[11]. By default, ICN approaches designed to support mobility, however, many challenges arose in some approaches like NDN in support of content mobility [12]-[15]. Hierarchical naming structure of NDN offers some benefits, such as route aggregation, improvement of scalability, multicasting. However, the naming structure causes significant challenge when content producer moves to a new location. Zhu et al. [4] ascertain the content consumer mobility support in NDN and content producer faces similar handoff challenges like an IP architecture. Moreover, suggested that content identifier and content locator need to be separated and mapped between them. Feng et al. [16]and Saxena et al. [17] surveyed general support of mobility in NDN, testified that content consumer mobility was integrally supported in NDN architecture, while content producer mobility faces many complications and challengesfundamentally. In addition, expresses the consumer mobility support in NDN by means of in-network caching and consumer-driven nature of NDN[16]. Therefore, some researches were carried out to improved consumer mobility using mobility link service [18], however, there are many problems such as, high handoff latency, high handoff signaling overhead cost, unnecessary Interest packets and poor data packets delivery that needs to be addressed. Fig 3 shows the how producer movement affected the seamless mobility in NDN.

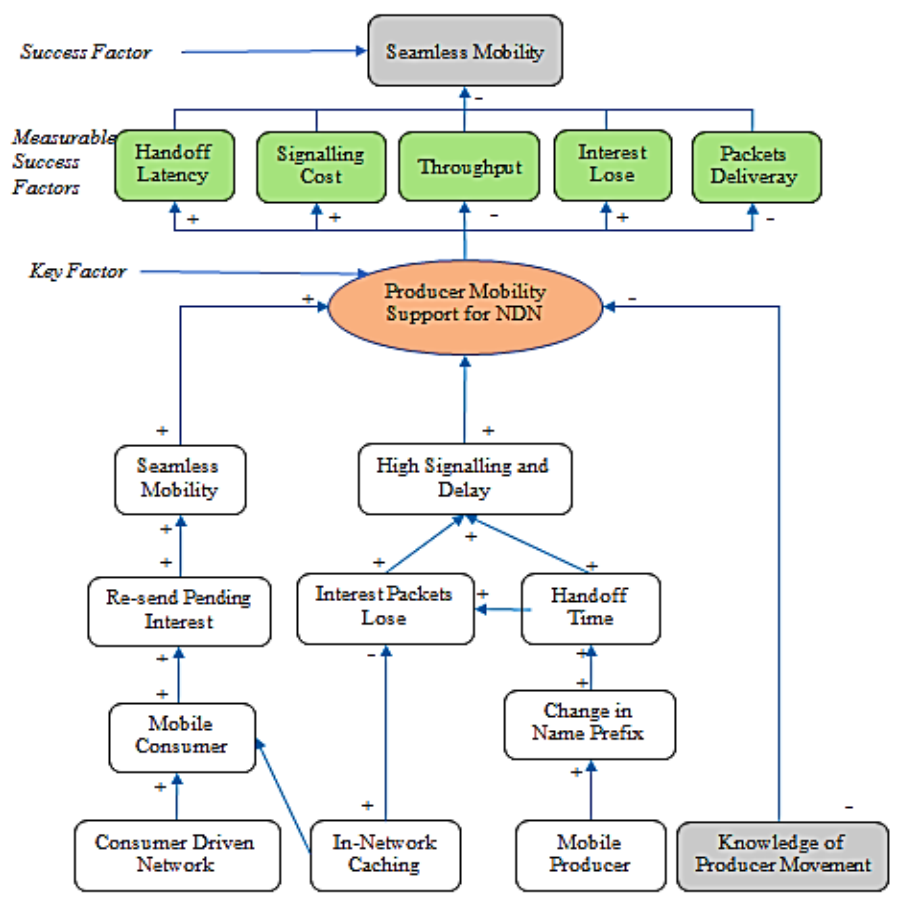

Fig. 3. Initial Reference Model of Seamless Mobility in NDN [19] 
Consumer mobility is supported due to the nature of in-network catching and consumer-driven nature of NDN[20], [21]. The catching suppressed unnecessary Interest packets losses and consumer-driven nature influencedthe mobile consumer to resed unsatisfied Interest packet immediately after the handoff, as shown in Fig 3. Once producer move to new location, automatically the prefix changed automatically after handoff to the network router of the point of attachment. The entire network lacks the knowledge of producer movement unless if the producer announces its new prefix to update the FIBs of intermediate routers. Lack of a producer's movement knowledge causes elongation of handoff latency and high-Interest packets losses. Resending Interests packets towards the old location of the producer intensify high signaling overhead and poor utilization of bandwidth and packets delivery. Therefore, there is needs to provide substantial producer mobility support to minimize the handoff latency, handoff signaling overhead cost, reduce the unnecessary Interest packets loss to improve data packets delivery once a content producer relocates to another PoA. Hence, this paper attempted to address the deficiency of Kite scheme to minimize handoff signaling cost and provide data path optimization after the handoff.

\section{$2 \quad$ Related Works}

Many researchers [22]-[25]made an effort to define, improved mobility management and schemes for NDN, to support either consumer or producer mobility. Kite mobility support was proposed by Zhang et al. [26], that uses two new features to support producer mobility in NDN, that is, locator-freeness and scenario-awareness. Moreover, Kite utilizes the state of PITs to trace and reached mobile nodes, also makes the new location of a mobile producer transparent through routable anchors [26]. This scheme is called a PIT-based approach [27] or anchor-based approach [28], where the mobile producer sends a traced Interest to the immobile anchor router, notifying it's new location and establish trace in each PIT tables along the intermediate routers to the immobile anchor. The corresponding node or immobile server requesting content sends an Interest to the immobile anchor that modified the Interest by adding a trace-name field and trace-only flag, which is called tracing Interest. The tracing Interest will be sent along the PIT trace to the current location of the producer. However, the Kite scheme outperforms the mapping-based approach in terms of handoff delay with similar signaling overhead when mobile nodes move frequently. In addition, the optimal path is not guaranteed in such a way that consumer's Interest packets always pass through the immobile anchor. Hence, Kite scheme fall into the category of indirection-based approach, that can make the forwarding path longer than the path computed in normal NDN routing plane.

Recent researches were proposed schemes to improve the performance of kite to minimize the handoff delay, signaling overhead cost and to provide optimal path[27], [28]. Do and Kim [27] proposed an optimal producer mobility support scheme for a large-scale NDN to reduce handoff latency, signaling overhead cost and to solve the sub-optimal routing of many schemes including Kite. The scheme proposed a mechanism to make the fast handoff and establishes an optimal path to direct next 
Interest packets to new PoA using special mobility management packet before handoff occurs. The NDN content routers at the domain border were modified to have the information about its neighbor to support the location of the producer. In addition, the scheme predicts the movement of producer and uses NDN forwarding plane state information to establish an optimal path but is not directly indirection-based approach. However, The author [27] claims that the solution is unrealistic for worldwide deployment due to the requirement of changing routers. Also, the scheme causes very high signaling on the processes of fast handoff and establishment of an optimal path before the handoff. Moreover, the additional of neighbor information and binding cache tables in the routers increases the look-up time during name prefix matching in addition to PIT and FIB tables.

Recently, Kim and Ko [28] proposed a producer mobility support scheme called on-demand anchor-based with the concept of indirection-based approach to improving upon Kite. The scheme utilizes adaptive Interest packets forwarding to provide the solution after the movement of the producer. When the FIB entries are invalid because of producer's handoff, the consumer's Interest packets would be redirected to the anchor node from the old PoA instead of being dropped. The anchor node used the mobility update sent by the mobile producer after the handoff to trace the new PoA and forwards the pending Interest toward the producer. The scheme was proposed to reduce time delay and network overhead caused by Interest fallback or blind flooding nature of Kite [28]. However, the scheme performs better by using Interest forwarding to minimizes packets transmission delay and overhead cost and shows stable performance when the speed of mobile nodes increases, but did not solve the suboptimal and triangular routing nature of Kite scheme. Moreover, the overall scheme result did not consider the handoff delay and its signaling overhead due to the handoff processes.

\section{The Concept of Proposed Scheme}

To provide producer mobility support in NDN, a content identifier and locator needs to be separated and coupled. The coupling can either be using a broadcasting method or intermediate servers [4]. NDN support Interest Broadcasting as the Interest are small in size making it feasible to be broadcasted in the restricted domain to track the location of mobile producer [4]. Asif and Kim [29] proposed a protocol Locationaware on-demand multipath for NDN mobile ad-hoc networks that used broadcast transmission mechanism for Interest packets or data. The packet follows multiple routes to locate the producer. A relay node was used to prevent intermittent connectivity, low battery power, data redundancy, packet flooding that affect network performance [29]. The author [29] compare or benchmark his research with similar protocols that utilize broadcast transmission mechanisms such as provider-aware forwarding and blind forwarding [30]. Further, Shi, Newberry and Zhang [31] study show how broadcast-based self-learning can be applied in NDN networks to solve name-prefix granularity and trust problem. In addition, broadcast-based self-learning is a common mechanism to find packets delivery path for mobility support [31]. 
Therefore, in this study broadcast method will be used to design a strategy that update intermediate routers with new mobility information of content producer when it's relocated to new PoA, in addition, restricted domain as in [4], [32] and distributed anchors as in [33] will be used to prevent scalability issue and guaranteed path optimization.

\subsection{Optimal broadcast strategy-based design}

In Named Data Networking, each mobile producer can have a namespace that represents its content name, at the same time serves as an identifier and locator for that content producer. However, there is no mechanism in NDN that can support and control the handoff process of mobile producer, to maintain the identifier and locator relationship. Therefore, we proposed an Optimal Broadcast Strategy-Based producer mobility support scheme to support, manage and control the handoff processes when content consumer relocates to another point of attachment. Conceptually, this paper introduced a Mobility Interest (MI) packet used to updates the binding information between previous and current access routers that content producer relocates. In addition, MI broadcasting strategy was designed to updatesthe intermediate routers and immobile anchor router is modified to manage broadcasting of MI packets to update the intermediate routers to make the optimal path. Fig. 4 shows the impact model of the proposed scheme using producer mobility update packets and broadcast strategy to provide knowledge of producer movement to support seamless mobility. The presence of the knowledge will reduce the handoff latency period, unnecessary Interest packet losses and signaling overhead cost. Moreover, increases overall throughput and packets delivery through the optimal path.

Consider Fig. 5, the network analysis model that shows the mode of operation for the proposed scheme and provision of the optimal route after the handoff. Initially, the consumer requested data content throughAR3 and AR1 to the static content producer by sending Interest packet, the producer sends the data to the consumer as normal. The content producer decided to move before completion of sending complete data content to the consumer, detects a strong signal and connected to the AR2. Immediately after the handoff, the content producer sends its new name prefix in MI packet through AR2 to the known location of anchor AR. The anchor router broadcast the MI packet to update the FIBs of the intermediate routers within the domain. When a consumer sends a pending Interest, AR3 will find the optimal route toward the AR2 to the content producer and replied data back. 
Paper-Optimal Broadcast Strategy-Based Producer Mobility Support Scheme for Named...

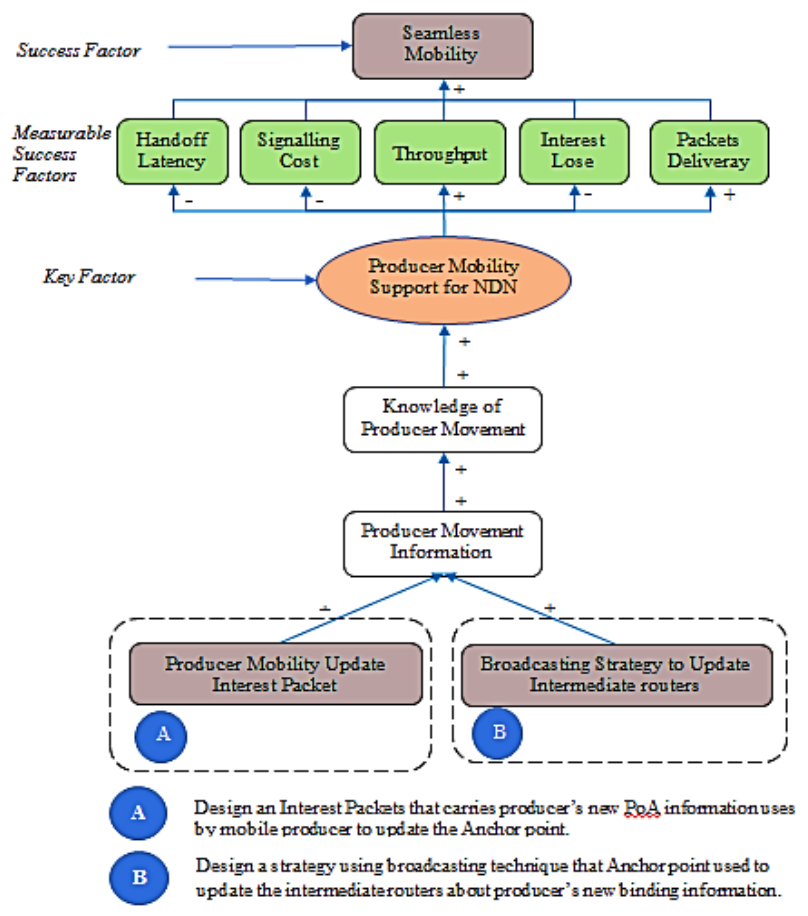

Fig. 4. Initial Impact Model [34]

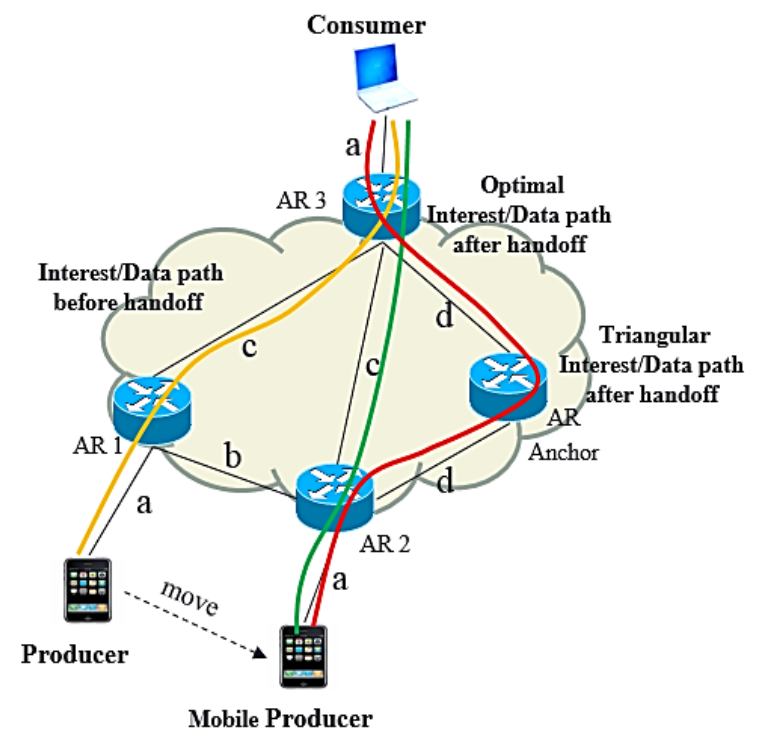

Fig. 5. Network Analysis Model 


\section{Analytical Investigation}

Predominantly, handoff problem were being investigated using the analytical method, whereas other issues like the determination of packets delivery, packets losses and scalability needs to be investigated using simulation for a better result. Henceforth, in this paper, network analysis model is used to formulate the mathematical models for the validation of proposed solution of producer mobility support concept to evaluate the handoff latency, signaling cost and routing path optimization. We consider network analysis model as done in [27], [35], [36], the random Waypoint Mobility model [37]-[40] for the movement behavior of the content producer as in (1). The total delay between two hops for wired and wireless link [27] shown in (2) and (3) Where $\mathrm{q}(0<=\mathrm{q}<=1)$ is the probability of link failure, $\mathrm{Bw}=100 \mathrm{Mbps}$ and Bowl = $11 \mathrm{Mbpsare}$ bandwidth for wired and wireless, Qd $=5$ msis the queuing delay, $\mathrm{Ldw}=2 \mathrm{~ms}$ and $\mathrm{Ldwl}=10 \mathrm{~ms}$ are wired and wireless link delay. The values of Sname are shown in Table 1.

Table 1. Network parameters and Values

\begin{tabular}{|c|c|c|c|}
\hline Category & Notation & Parameters & Value \\
\hline \multirow[t]{5}{*}{ General } & $S_{\text {name }}$ & Size of the signaling packet & 72 byte \\
\hline & $S_{\text {data }}$ & Size of data packets & 1024 bytes \\
\hline & $S_{\text {iint }}$ & Size of Interest packet & 56 bytes \\
\hline & $V_{c p}$ & Average speed of content producer & $50 \mathrm{~m} / \mathrm{s}$ \\
\hline & $t_{p}$ & Content producer pause time & $100 \mathrm{~ms}$ \\
\hline \multirow[t]{7}{*}{ Latency } & $L_{p a r}$ & Transmission latency between producer to AR & $a$ \\
\hline & $L_{c a r}$ & Transmission latency between consumer to AR & $a$ \\
\hline & $L_{\text {sar }}$ & Transmission latency between old AR to new AR & $b$ \\
\hline & $L_{o-n a r}$ & Transmission latency between Ars & $c$ \\
\hline & Lars & Transmission latency between ARs and Anchors & $d$ \\
\hline & $I_{p n}$ & $\begin{array}{l}\text { Time interval btw producer disconnection and } \\
\text { reconnection from old AR to new AR }\end{array}$ & $i_{p n}$ \\
\hline & $Q$ & The probability of link failure & $q$ \\
\hline \multirow[t]{7}{*}{ Signaling Cost } & $C_{p a r}$ & Transmission cost hop/packet producer to AR & $a$ \\
\hline & $C_{c a r}$ & Transmission cost hop/packet consumer to AR & $a$ \\
\hline & $C_{o-n a r}$ & Transmission cost hop/packet old AR to new AR & $b$ \\
\hline & $C_{a r s}$ & Transmission cost hop/packet Ars & $c$ \\
\hline & $C_{s-n a r}$ & Transmission cost hop/packet AR to Anchors & $d$ \\
\hline & $I A R$ & Interest arrival rate & $\gamma$ \\
\hline & $S C R$ & Subnet crossing rate & $\mu$ \\
\hline
\end{tabular}

$$
\begin{gathered}
\text { Mobility }=\left(1-\left(\frac{t_{p}}{t_{p}+(0.3 \times v)}\right)\right) \\
L w_{\text {name }}=\left(\frac{S_{\text {name }}}{(B w+L d w+Q d)}\right) \\
L w l_{\text {name }}=\left(\frac{1+q}{1-q}\right) \times\left(\frac{S_{\text {name }}}{B w l+L d w l}\right)
\end{gathered}
$$




\subsection{Handoff Latency Analysis}

Handoff or handover refers to the processes where a mobile content producer disconnects and relocates from current AR or Access Point (AP) and connects to next $\mathrm{AR}$ or AP. The period of time required for a mobile producer to complete the signaling processes of disconnection from the current $\mathrm{AR}$ or AP and reconnection to the new AR or PA is called handoff latency. The latency model is formulated using hop count parameters for transmission latency between hops $(a, b, c, d)$, RWP model [40] movement behavior (1) in the area of $[0, \mathrm{~A}]^{2}$ and the link delay between two consecutive hops (2) and (3). The handoff latency is formulated for our proposed scheme optimal broadcast strategy-based (OBSB) and other two schemes to compared and benchmark the performance of our scheme. The two schemes are Kite [22], [26] and optimal provider mobility support in large-scale NDN (OPML) [27], the models were shown in (4), (5) and (6) for OPML, Kite, and OBSB respectively.

$$
\begin{gathered}
L_{\text {opml }}=\left(1-\left(\frac{t_{p}}{t_{p}+(0.3 \times v)}\right)\right) \times \\
\left(\frac{l_{p n}+a \times L w l_{\text {qryRep }}+a \times L w l_{\text {fasthanf }}+3 c \times L w_{\text {opt_del_makroute }}}{A^{2}}\right) \times\left(\frac{+2 a \times L w l_{\text {ackInt }}+c \times L w_{\text {int }}}{A^{2}}\right) \\
L_{\text {kite }}=\left(1-\left(\frac{t_{p}}{t_{p}+(0.3 \times v)}\right)\right) \times \\
\left(\frac{l_{\text {pn }}+a \times L w l_{\text {up }}+2 d \times L w_{\text {trcgInt }}+a \times L w l_{\text {trcdInt }}+2 d \times L w_{\text {trcdInt }}}{A^{2}}\right) \\
L_{o b s b}=\left(1-\left(\frac{t_{p}}{t_{p}+(0.3 \times v)}\right)\right) \times\left(\frac{l_{p n}+a \times L w l_{n i n t}+2 d \times L w l_{\text {nint }}+a \times L w l_{\text {int }}+c \times L w_{\text {int }}}{A^{2}}\right)
\end{gathered}
$$

The content producer in OPML processed a fast handoff before disconnecting and relocation to new AR by sending a neighbor query to the AR1 and replies, and then sends fast handoff to AR1. The AR1 send a message to create an optimal path by making a new route between AR3 and AR2 and delete the existing route between AR3 and AR1 after that handoff takes place. In Kite, after the handoff processes, content producer send a traced Interest from AR2 to the known location of immobile anchor router. The corresponding node or consumer sends a tracing Interest from AR3 to the anchor, where it will be redirected to the new location of the content producer. The cumulative delay for these signaling corresponding to facilitate the handoff processes are considered as handoff latency, the smaller the latency the better performance of the scheme.

\subsection{Handoff signaling cost analysis}

The handoff cost is the total number of messages or packets sent over the period time of handoff processes when mobile producer disconnected from the current AR and reconnected to the new AR or PA. The signaling cost model is formulated using hop count parameters for transmission cost per packets between hops $(a, b, c, d)$, RWP model movement behavior (1) and handoff related packets size $\left(S_{\text {name }}\right)$ and 
Interest arrival rate $(\gamma)$. The handoff signaling cost is formulated for our proposed scheme and other two benchmark schemes were shown in (7), (8) and (9) for OPML, Kite and OBSB respectively.

$$
\begin{aligned}
& C_{\text {opml }}=\gamma \times\left(1-\left(\frac{t_{p}}{t_{p}+(0.3 \times v)}\right)\right) \times\left(\frac{S_{\text {qryrep }} \times(2 a)+S_{\text {optRoute }}(3 c)+S_{\text {int }} \times(a+c)}{A^{2}}\right) \\
& C_{\text {kite }}=\gamma \times\left(1-\left(\frac{t_{p}}{t_{p}+(0.3 \times v)}\right)\right) \times\left(\frac{S_{\text {trcInt }} \times(a+2 d)+S_{\text {int }} \times(a+2 d)}{A^{2}}\right) \\
& C_{p m s s}=\gamma \times\left(1-\left(\frac{t_{p}}{t_{p}+(0.3 \times v)}\right)\right) \times\left(\frac{S_{\text {mobInt }} \times(a+2 d)+S_{\text {int }} \times(a+c)}{A^{2}}\right)
\end{aligned}
$$

The content producer in OPML send a fast handoff, neighbor query, optimal path make and delete route messages or packets between producer, AR1, and AR3 before handoff takes place. In Kite, after the handoff processes, content producer exchanges traced and tracing Interests between producer, immobile anchor router and corresponding node or consumer. The proposed scheme sent MI packets to AR2, AR2 to the anchor and broadcast to AR3 The cumulative signaling messages correspond to facilitate the handoff processes are considered as handoff signaling cost, the smaller the cost the better performance of the scheme.

\subsection{Packets delivery analysis}

The packets delivery cost is the total number of hope per packets sent over after the handoff processes from mobile producer to the content consumer. The packets delivery cost model is formulated using hop count parameters for transmission cost per packets between hops ( $a, b, c, d)$, RWP model movement behavior (1) and handoff related packets size $\left(S_{\text {name }}\right)$ and subnet crossing rate $(\mu)$. The model is formulated for our proposed scheme and other two benchmark schemes were shown in (10), (11) and (12) for OPML, Kite and OBSB respectively.

$$
\begin{aligned}
& C_{\text {opml }}=\mu \times\left(1-\left(\frac{t_{p}}{t_{p}+(0.3 \times v)}\right)\right) \times\left(\frac{S_{\text {int }} \times(2 a+c)+S_{\text {data }} \times(2 a+c)}{A^{2}}\right) \\
& C_{\text {kite }}=\mu \times\left(1-\left(\frac{t_{p}}{t_{p}+(0.3 \times v)}\right)\right) \times\left(\frac{S_{\text {int }} \times(2 a+2 d)+S_{\text {data }} \times(2 a+2 d)}{A^{2}}\right) \\
& C_{o b s b}=\mu \times\left(1-\left(\frac{t_{p}}{t_{p}+(0.3 \times v)}\right)\right) \times\left(\frac{S_{i n t} \times(2 a+c)+S_{\text {data }} \times(2 a+c)}{A^{2}}\right)
\end{aligned}
$$

After the handoff process in OPML, the route is created from consumer to mobile producer through AR3 and AR2. The same route created in the proposed scheme, but different in Kite. The shortest path is the optimal data path. 


\section{$5 \quad$ Numerical Result}

\subsection{Handoff latency}

The summary of the numerical result for handoff latency is shown in Table 2 and Fig 6 depicts the impact of wireless link failure. We can observe that our proposed scheme (OBSB) and Kite have almost the same handoff latency as the probability of link failure increases.

The handoff latency of OPML is higher and keeps increasing as link failure probability getting higher. From Table 2 we can see that at $q=0.8$ onward, the latency of OPML is getting twice than Kite and OBSB. Although optimal data path may play a role to reduce latency, the OPML provide an optimal path, but a large number of signaling messages took place as fast handoff, route making, and deletion processes.

We can conclude that our proposed scheme performed well than OPML and no significant improvement on Kite scheme in terms of handoff latency.

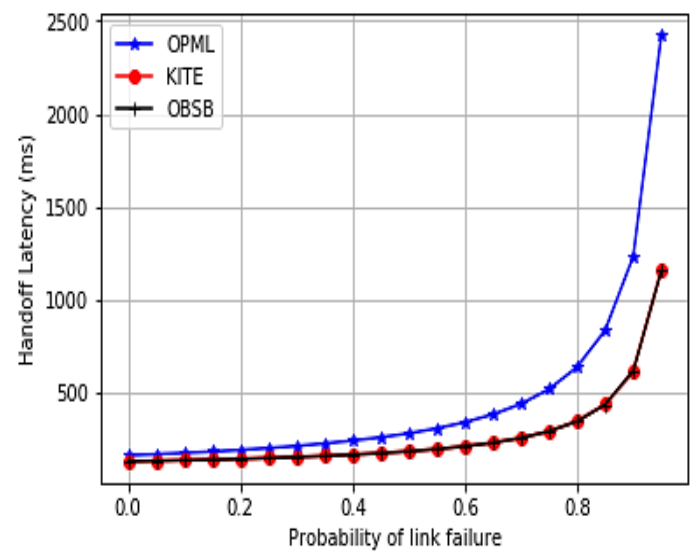

Fig. 6. Handoff Latency against Link Failure Probability

Table 2. Result Comparison of Handoff Latency

\begin{tabular}{|l|c|c|c|c|c|c|c|c|}
\hline \multicolumn{1}{|c|}{ Probability (q) } & $\mathbf{0}$ & $\mathbf{0 . 2}$ & $\mathbf{0 . 4}$ & $\mathbf{0 . 6}$ & $\mathbf{0 . 8}$ & $\mathbf{0 . 8 5}$ & $\mathbf{0 . 9}$ & $\mathbf{0 . 9 5}$ \\
\hline OPML & 161 & 190 & 240 & 339 & 636 & 835 & 1231 & 2421 \\
\hline KITE & 129 & 143 & 165 & 210 & 345 & 435 & 616 & 1156 \\
\hline OBSB & 128 & 141 & 164 & 209 & 344 & 434 & 614 & 1154 \\
\hline
\end{tabular}

\subsection{Handoff signaling cost}

The summary of the numerical result for handoff signaling cost is shown in Table 3 and in Fig. 7we compared the performance of our proposed scheme with OPML and Kite. The handoff signaling cost is presented with the variation of Interest arrival rate. 
The handoff signaling cost of OPML is significantly high and keeps increasing as the Interest arrival rate increase, due to the fast handoff signaling messages, while the signaling cost of Kite is less than OPML and higher than OBSB. We can infer that our proposed scheme (OBSB) has better handoff cost with significant improvement on Kite and OPML as the arrival rate of Interest increases.

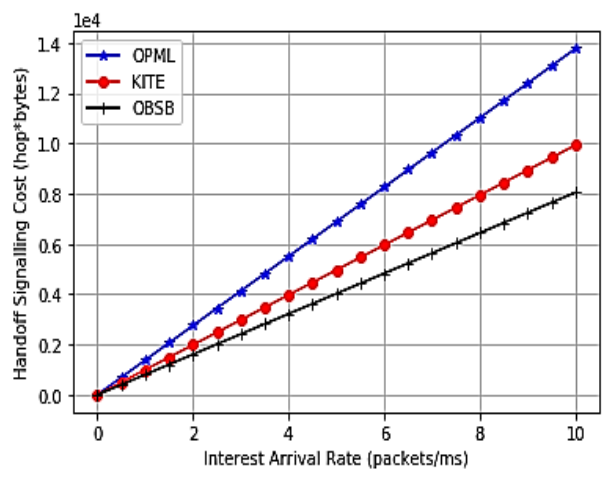

Fig. 7. Handoff Signaling Cost against Interest Arrival Rate

Table 3. Result Comparison of Handoff Signaling Cost

\begin{tabular}{|l|c|c|c|c|c|c|c|}
\hline \multicolumn{1}{|c|}{ Interest Arrival Rate $(\boldsymbol{\gamma})$} & $\mathbf{1}$ & $\mathbf{3}$ & $\mathbf{5}$ & $\mathbf{7}$ & $\mathbf{9}$ & $\mathbf{9 . 5}$ & $\mathbf{1 0}$ \\
\hline OPML & 1376 & 4128 & 6881 & 9633 & 12385 & 13074 & 13762 \\
\hline KITE & 993 & 2978 & 4963 & 6948 & 8934 & 9430 & 9926 \\
\hline OBSB & 805 & 2414 & 4023 & 5632 & 7242 & 7644 & 8046 \\
\hline
\end{tabular}

\subsection{Packets delivery cost}

The packets delivery cost determine the data path optimization, Fig. 8 shows the packets delivery cost performance of our proposed scheme compared with Kite and OPML, where Table 4 highlight the summary of the numerical result. From Fig 8 we can see that our scheme and OPML coincide due to the provision of optimal data path after the handoff. From Table 4 we can see that OBSB and OPML have the same value as subnet crossing net increases. The packets delivery cost of Kite increases significantly because Interest and data packets most passed through the anchor point creating sub-optimal or triangular routing. Therefore, our scheme provides data path optimization after the handoff completion, hence improved upon Kite scheme. 


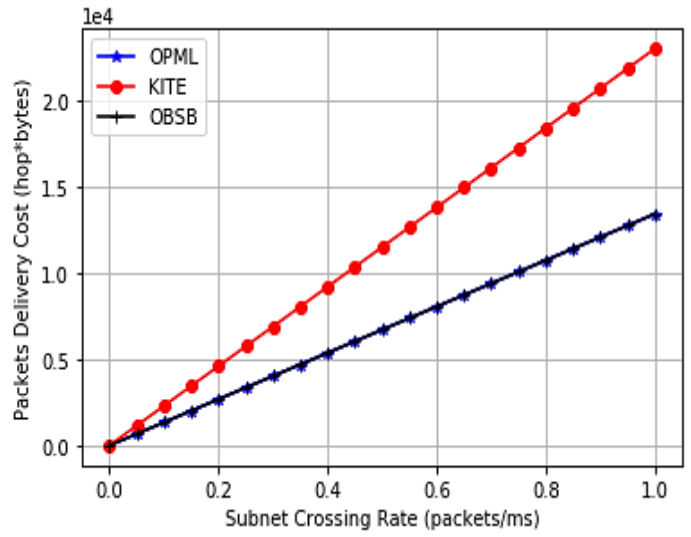

Fig. 8. Optimal Packets Delivery Cost against Subnet Crossing Rate

Table 4. Result Comparison of Packets Delivery Cost

\begin{tabular}{|l|c|c|c|c|c|c|c|}
\hline Subnet Crossing Rate & $\mathbf{0 . 1}$ & $\mathbf{0 . 3}$ & $\mathbf{0 . 5}$ & $\mathbf{0 . 7}$ & $\mathbf{0 . 9}$ & $\mathbf{0 . 9 5}$ & $\mathbf{1}$ \\
\hline OPML & 1342 & 4027 & 6712 & 9396 & 12081 & 12752 & 13423 \\
\hline KITE & 2301 & 6903 & 11506 & 16108 & 20710 & 21861 & 23011 \\
\hline OBSB & 1342 & 4027 & 6712 & 9396 & 12081 & 12752 & 13423 \\
\hline
\end{tabular}

\section{Conclusion}

In Named Data Networking architecture there is needs to provide a solution to the producer mobility support problems, to minimize handoff latency, handoff signaling overhead cost, improve data packets delivery through optimal route and minimizes the unnecessary Interest packets losses once a content producer relocates to another access point. In this paper, an optimal broadcast strategy-based scheme was proposed to facilitate and support the handoff processes by updating the intermediate routers FIBs about the producer's movement. Hence, the performance analysis of the proposed scheme was analytically investigated, the result addressed the data path optimization deficiency of Kite scheme. The numerical result shows that our proposed OBSB scheme outperforms OPML with less handoff signaling cost and latency. In addition, reduces handoff signaling cost and provides optimal data path compared to Kite. Henceforth, in our future work, we will put more emphases on simulation investigation to ascertain the data path optimization in a large simulated environment.

\section{$7 \quad$ References}

[1] L. Zhang et al., "Named Data Networking," ACM SIGCOMM Comput. Commun. Rev., vol. 44, no. 3, pp. 66-73, 2014. https://doi.org/10.1145/2656877.2656887

[2] L. Zhang et al., "Named Data Networking," NDN, Tech. Rep. NDN-0001, no. October, 2010. 
[3] A. Dowling, M. Babaeianjelodar, Y. Liu, and K. Chen, "Performance evaluation of NDN applications in low-interference Mobile Ad Hoc Environments," in 2018 IEEE International Conference on Communications, 2018, pp. 1-6. https://doi.org/10.11 09/ICC.2018.8422756

[4] Z. Zhu, A. Afanasyev, and L. Zhang, "A new perspective on mobility support," NamedData Networking Project, Technical Report NDN-0013, pp. 1-6, 2013.

[5] S. Mastorakis, A. Afanasyev, and L. Zhang, "On the Evolution of ndnSIM," ACM SIGCOMM Comput. Commun. Rev., vol. 47, no. 3, pp. 19-33, 2017. https://doi.org/10.1 $145 / 3138808.3138812$

[6] S. Mastorakis, A. Afanasyev, I. Moiseenko, and L. Zhang, "ndnSIM 2 : An updated NDN simulator for NS-3," NDN, Tech. Rep. NDN-0028, 2015, pp. 1-8, 2016.

[7] M. Hussaini, S. A. Nor, A. Ahmad, I. S. Maijama'a, A. Isah, and A. Aminu, "Mobility support and operation of Information Centric Networking approach," Int. J. Comput. Netw. Inf. Secur., vol. 10, no. 10, pp. 18-25, 2018. https://doi.org/10.5815/ijcnis.2018.10.03

[8] D. Liu, C. Huang, X. Chen, and X. Jia, "Space-terrestrial integrated mobility management via Named Data Networking," Tsinghua Sci. Technol., vol. 23, no. 4, pp. 431-439, 2018. https://doi.org/10.26599/TST.2018.9010042

[9] M. Gohar, N. Khan, A. Ahmad, M. Najam-ul-islam, S. Sarwar, and S. Koh, "Cluster-based device mobility management in Named Data Networking for Vehicular Networks," Mob. Inf. Syst., vol. 2018, pp. 1-7, 2018.

[10] J. M. Duarte, T. Braun, and L. A. Villas, "MobiVNDN: A distributed framework to support mobility in vehicular named-data networking," Ad Hoc Networks, vol. 82, pp. 7790, 2019. https://doi.org/10.1016/j.adhoc.2018.08.008

[11] L. Rui, S. Yang, and H. Huang, "A producer mobility support scheme for real-time multimedia delivery in Named Data Networking," Multimed. Tools Appl., vol. 77, no. 4, pp. 4811-4826, 2018. https://doi.org/10.1007/s11042-017-5601-1

[12] M. Hussaini, S. A. Nor, and A. Ahmad, "Producer mobility support for Information Centric Networking approaches: A review," Int. J. Appl. Eng. Res., vol. 13, no. 6, pp. 3272-3280, 2018

[13] D. Kutscher et al., "Information-Centric Networking (ICN) Research Challenges," Internet Research Task Force (IRTF) Request for Comments: 7927, pp. 1-38, 2016.

[14] C. Fang, H. Yao, Z. Wang, W. Wu, X. Jin, and F. R. Yu, "A survey of mobile InformationCentric Networking: Research issues and challenges," IEEE Commun. Surv. Tutorials, vol. 20, no. 3, pp. 2353-2371, 2018. https://doi.org/10.1109/COMST.2018.2809670

[15] M. Hussaini, S. A. Nor, and A. Ahmad, "Producer mobility support scheme for Named Data Networking: A survey,” Int. J. Electr. Comput. Eng., vol. 8, no. 6, pp. 31-42, 2018. https://doi.org/10.11591/ijece.v8i6.pp5432-5442

[16] B. Feng, H. Zhou, and Q. Xu, "Mobility support in Named Data Networking: a survey," Eurasip J. Wirel. Commun. Netw., vol. 2016, no. 1, 2016. https://doi.org/10.1186/s13638016-0715-0

[17] D. Saxena, V. Raychoudhury, N. Suri, C. Becker, and J. Cao, "Named Data Networking: A Survey," Comput. Sci. Rev. Elsevier, vol. 19, pp. 15--55, 2016. https://doi.org/10.1016/j.cosrev.2016.01.001

[18] J. Cha, J. Choi, J. Kim, Y. Han, and S. Min, "A Mobility Link Service for NDN Consumer Mobility,” Wirel. Commun. Mob. Comput., vol. 2018, pp. 1-8, 2018. https://doi.org/10.1155/2018/5149724

[19] L. Blessing and A. Chakrabarti, "DRM: A Design Research Methodology," in In proceedings of Les Science de la Conception, 2002, pp. 1-15.

[20] I. Abdullahi, S. Arif, and S. Hassan, "Survey on caching approaches in Information Centric Networking," J. Netw. Comput. Appl., vol. 56, no. July, pp. 48-59, 2015. https://doi.org/10.1016/j.jnca.2015.06.011

[21] . U. Din, S. Hassan, M. K. Khan, M. Guizani, O. Ghazali, and A. Habbal, "Caching in Information-Centric Networking: Strategies, challenges, and future research directions," IEEE Commun. Surv. Tutorials, vol. 20, no. 2, pp. 1443-1474, 2018. https://doi.org/10.1109/COMST.2017.2787609 
[22] Y. Zhang, H. Zhang, and L. Zhang, "Kite: A Mobility Support Scheme for NDN," in Proceedings of the 1st ACM Conference on Information-Centric Networking- (ICN) ACM-ICN '14, 2014, no. 1, pp. 179-180. https://doi.org/10.1145/2660129.2660159

[23] J. Augé, G. Carofiglio, G. Grassi, L. Muscariello, G. Pau, and X. Zeng, "MAP-Me: Managing anchor-less producer mobility in Information-Centric Networks," IEEE Trans. Netw. Serv. Manag., vol. 15, no. 2, pp. 596-610, 2018. https://doi.org/10.1 109/TNSM.2018.2796720

[24] M. Hussaini, S. A. Nor, and A. Ahmad, "PMSS : Producer mobility support scheme optimization with RWP mobility model in Named Data Networking," Int. J. Commun. Networks Inf. Secur., vol. 10, no. 2, pp. 329-339, 2018.

[25] M. Meddeb, A. Dhraief, A. Belghith, T. Monteil, K. Drira, and S. Gannouni, "AFIRM: Adaptive forwarding based link recovery for mobility support in NDN/IoT networks," Futur. Gener. Comput. Syst., vol. 87, pp. 351-363, 2018. https://doi.org/10.1 016/j.future.2018.04.087

[26] Y. Zhang, H. Zhang, and L. Zhang, “Kite,” NDN, Technical Report NDN-0020, 2014., no. 1, NDN Project, pp. 1-10, 2014.

[27] T. X. Do and Y. Kim, "Optimal provider mobility in large-scale Named- Data Networking,” KSII Trans. Internet Inf. Syst., vol. 9, no. 10, pp. 4054-4071, 2015.

[28] D. Kim and Y. Ko, "On-demand anchor-based mobility support method for Named Data Networking," in 19th International Conference on Advanced Communication Technology (ICACT), 2017, 2017, pp. 19-23. https://doi.org/10.23919/ICACT.2017.7890049

[29] R. A. Rehman and B.-S. Kim, "LOMCF: Forwarding and Caching in Named Data Networking-based MANETs," IEEE Trans. Veh. Technol., vol. 66, no. 10, pp. 1-1, 2017. https://doi.org/10.1109/TVT.2017.2700335

[30] M. Amadeo, C. Campolo, and A. Molinaro, "Forwarding strategies in named data wireless ad hoc networks: Design and evaluation," J. Netw. Comput. Appl., vol. 50, pp. 148-158, 2015. https://doi.org/10.1016/i.jnca.2014.06.007

[31] J. Shi and E. Newberry, "On Broadcast-based Self-Learning in Named Data Networking," in IFIP Networking, 2017, pp. 1-9.

[32] D. Kim, J. Kim, Y. Kim, H. Yoon, and I. Yeom, "End-to-end mobility support in Content Centric Networks," Int. J. Commun. Syst., vol. 28, no. 6, pp. 1151-1167, Apr. 2015. https://doi.org/10.1002/dac.2752

[33] A. Azgin, R. Ravindran, and G. Wang, "On-demand mobility support with anchor chains in Information Centric Networks," IEEE Int. Conf. Commun., 2017. https://doi.org/10.1 109/ICC.2017.7997210

[34] R. Alubady, M. Al-samman, S. Hassan, S. Arif, and A. Habbal, "Internet Protocol MANET vs Named Data MANET : A Critical Evaluation," 4th Int. Conf. Internet Appl. Protoc. Serv. Internet, no. July 2016, pp. 70-76, 2015.

[35] S. Gao and H. Zhang, "Scalable mobility management for content sources in Named Data Networking," 2016 13th IEEE Annu. Consum. Commun. Netw. Conf. CCNC 2016, pp. 79-84, 2016.

[36] Y. Rao, H. Luo, D. Gao, H. Zhou, and H. Zhang, "LBMA: A novel Locator Based Mobility support Approach in Named Data Networking," China Commun., vol. 11, no. 4, pp. 111-120, 2014. https://doi.org/10.1109/CC.2014.6827573

[37] L. Guezouli, K. Barka, S. Bouam, and A. Zidani, "Implementation and optimization of RWP mobility model in WSNs under TOSSIM simulator," Int. J. Commun. Networks Inf. Secur., vol. 9, no. 1, pp. 1-11, 2017.

[38] C. Bettstetter, H. Hartenstein, and X. Pérez-Costa, "Stochastic properties of the random waypoint mobility model," Proc. 5th ACM Int. Work. Model. Anal. Simul. Wirel. Mob. Syst. - MSWiM '02, p. 7, 2002. https://doi.org/10.1145/570758.570761

[39] S.V. Manikanthan, T.Padmapriya, "United Approach in Authorized and Unauthorized Groups in LTE-A Pro", Jour of Adv Research in Dynamical \& Control Systems, Vol. 10, 10-Special Issue, 2018, pp. (1137-1145).

[40] C. Bettstetter, G. Resta, and P. Santi, "The node distribution of the random waypoint mobility model for wireless ad hoc networks," IEEE Trans. Mob. Comput., vol. 2, no. 3, pp. 257-269, 2003. https://doi.org/10.1109/TMC.2003.1233531 


\section{Authors}

Muktar Hussaini obtained the B.Sc. degrees in Computer Science from the Usman DanfodiyoUniversity Sokoto, Nigeria, in 2005, M.Sc.degree in Computer and Information Engineering, at International Islamic University Malaysia, in 2012. He is currently pursuing the Ph.D. degree in Computer Science at Universiti Utara Malaysia (UUM) Malaysia. He is currently a research student under the Internetwork Research Laboratory, School of Computing, UUM, also a member of Internet Society and Nigerian Computer Society. From 2006, he worked with the Department of Computer Science at Hussaini Adamu Federal Polytechnic, Kazaure Jigawa State, Nigeria. His research interest includes the energy efficiency of wireless sensor networking, wireless sensor protocol and applications, future Internet research, Information Centric networking (ICN), Named Data networking (NDN), mobility support for ICN and producer mobility support for NDN.

Shahrudin Awang Nor received the B.Eng. degree (Hons.) in electronics engineering from Universiti Sains Malaysia, the M.Sc. degree in computer and information networks from the University of Essex, U.K., and the Ph.D. degree in computer networks from Universiti Utara Malaysia (UUM). He is currently a Senior Lecturer and the Head of platform at the InterNetWorks Research Lab, School of Computing, College of Arts and Sciences, UUM. Also, a member IEEE and member Internet Society. His research interests include computer network and security, communication and network performance, network transport protocol, and computer organization.

Amran Ahmad obtained the B.Sc, in Information Technology (1995), M.Sc. in Information Technology (2003), and PhD degree in Information Technology (2015) at Universiti Utara Malaysia (UUM), Malaysia. Now is currently a Head of Department Computer Science and a Senior Lecturer at School of Computing, UUM. Also, a researcher with the InterNetWorks Research Laboratory, College of Arts and Sciences, UUM, a member IEEE and Internet Society. His research interest includes security services (Including digital forensic, steganography, network security, public key infrastructure and biometric) e-Learning, wireless network security, network management and web application development.

Article submitted 2019-01-08. Resubmitted 2019-02-27. Final acceptance 2019-03-05. Final version published as submitted by the authors. 